\title{
La force au féminin dans le conte merveilleux fin- de-siècle
}

\section{Michel Viegnes}

\section{(2) OpenEdition}

1 Journals

Édition électronique

URL : http://journals.openedition.org/edl/214

DOI : $10.4000 /$ edl. 214

ISSN : 2296-5084

Éditeur

Université de Lausanne

\section{Édition imprimée}

Date de publication : 15 décembre 2011

Pagination : 321-336

ISBN : 978-2-940331-26-0

ISSN : 0014-2026

\section{Référence électronique}

Michel Viegnes, "La force au féminin dans le conte merveilleux fin-de-siècle », Études de lettres [En ligne], 3-4 | 2011, mis en ligne le 15 décembre 2014, consulté le 19 décembre 2020. URL : http:// journals.openedition.org/edl/214; DOI : https://doi.org/10.4000/edl.214 


\section{LA FORCE AU FÉMININ \\ DANS LE CONTE MERVEILLEUX FIN-DE-SIÈCLE}

Dans plusieurs pays d'Europe, et en France en particulier, les deux dernières décennies du XIX $\mathrm{X}^{\mathrm{e}}$ siècle présentent un paradoxe bien connu: alors que triomphe le positivisme, la littérature et les arts, pour une large part, se tournent vers des mondes imaginaires souvent marqués par l'esthétique décadente du pervers et du morbide. Le genre du conte merveilleux, et même plus spécifiquement du conte de fées est remis à l'honneur par des poètes tels que Théodore de Banville, Catulle Mendès, Henri de Régnier, et des prosateurs comme Marcel Schwob. Sous la forme de la fée, de la femme-enfant ou de la princesse faussement ingénue, le personnage féminin s'y montre souvent redoutable, et presque toujours détenteur d'un savoir ou de facultés qui transforment les soi-disant représentantes du sexe faible en «femmes fatales» ambivalentes, simultanément fées et démones, muses à la fois séduisantes, étranges et vaguement menaçantes.

Pour l'homme du XIX $\mathrm{X}^{\mathrm{e}}$ siècle, la femme résume tout à la fois l'attrait et le vertige angoissant de l'altérité. Pour les écrivains et les artistes de ce siècle, qui sont plus de neuf fois sur dix des hommes, le féminin est l'Autre par excellence, et son étrangeté à la fois inquiétante et séduisante doit être exorcisée de deux manières: l'angélisation ou la diabolisation. Le premier procédé permet une sublimation de son pouvoir érotique; la créature éthérée fait moins peur, son piédestal la tient à distance respectueuse d'un réel où elle pourrait prendre trop de place. Le procédé inverse est la sanction symbolique de la femme qui veut devenir un sujet libre, qui entend sortir de sa définition purement sexuée pour entrer dans la dimension de l'humain, avec ses contradictions. Les «diaboliques» de Barbey d'Aurevilly, un terme qu'il faut entendre au féminin dans le titre 
de son recueil ${ }^{1}$, sont parfois perverses, mais le plus souvent elles ne sont que les victimes d'un ordre masculin, contre lequel elles retournent leurs armes favorites.

Dans les dernières décennies du siècle, alors que le discours scientifique, avec Charcot et Lumbroso, vient parfois apporter sa caution à la misogynie, en présentant la femme comme moins rationnelle que l'homme et en l'enfermant dans un déterminisme biologique, la culture décadentiste développe une mythologie imprégnée de gynophobie ${ }^{2}$. Le mépris misogyne y fait place à la peur, ou du moins à la crainte, devant l'affinité profonde entre le féminin et un monde invisible, ténébreux et fascinant. Quand ce mysterium tremendum ${ }^{3}$ du féminin ne s'incarne pas dans une prêtresse démoniaque telle que la Chantelouve de J.-K. Huysmans ${ }^{4}$, ou une esthète sadique comme la Clara du Jardin des supplices de Mirbeau ${ }^{5}$, il suscite une figure semi-légendaire, telle la Salomé de l'Evangile, qui devient le grand mythe fin-de-siècle dans la culture européenne ${ }^{6}$, ou bien un être surnaturel, notamment la fée; la renaissance spectaculaire du conte merveilleux s'explique en partie par l'ambivalence traditionnelle de la fée, bonne ou mauvaise, parfois bonne et mauvaise. Jean de Palacio, dans une étude désormais classique ${ }^{7}$, a analysé en détail ces "perversions du merveilleux" à la fin du siècle; nous reviendrons ici sur certains de ces motifs, et notamment sur celui du pouvoir féminin, à travers trois auteurs, Marcel Schwob, Catulle Mendès et Jean Lorrain ${ }^{8}$.

I. J. Barbey d'Aurevilly, Les Diaboliques.

2. Cf. mon article "Gynophobia».

3. Rudolf Otto, dans son étude fondatrice et classique sur le sacré publiée en 1917, mentionne ces deux faces inséparables du sentiment religieux primitif que sont le mysterium tremendum et le mysterium fascinans, ambivalence que l'on reconnaît dans ces figures féminines fin-de-siècle.

4. J. K. Huysmans, Là-bas, publié pour la première fois en 1891.

5. O. Mirbeau, Le Jardin des supplices.

6. Voir à ce sujet l'article de M. Décaudin, «Salomé».

7. J. de Palacio, Les Perversions du merveilleux.

8. Marcel Schwob (1867-1905), écrivain érudit et polygraphe, traducteur de Defoe (Moll Flanders), auteur de contes (Cour double, 1891, Le Roi au masque d'or, 1895), figure importante du Paris littéraire fin-de-siècle; Catulle Mendès (1841-1909), écrivain et journaliste, gendre de Théophile Gautier dont il avait épousé la fille Judith, elle-même écrivain, admirateur de Wagner dont il entretint le culte dans les années 1880; Jean Lorrain (1855-1906), véritable personnification du dandy fin-de-siècle, entre 
On peut distinguer quatre mythèmes présents dans la construction du personnage féminin chez Schwob: la supériorité dominatrice de la femme; son caractère intrinsèquement énigmatique, forclos; sa propension à fuir dans un univers imaginaire quasi solipsiste; son amoralité, qui la situe "par-delà le bien et le mal» 9 . L'un des contes qui composent le premier recueil de Schwob, Cour double (1891), s'intitule "Lilith", du nom d'une figure parabiblique qui constitue pour lui, et sans doute pour toute sa génération, le mythe fondateur par excellence du féminin. Le protagoniste, un poète en quête de l'Ewigeweibliche, s'attache à des femmes qui s'éloignent de plus en plus d'un type dominable et soumis. La courtisane Jenny est voluptueuse mais insensible, et ne s'intéresse qu'à son argent; sa seconde maîtresse est plus originale: «il choisit Hélène, qui tournait dans une poêle d'airain l'image en cire de son fiancé perfide: il l'aima, tandis qu'elle lui perçait le cœur avec sa fine aiguille d'acier ${ }^{10}$; lassé de ces délices magico-masochistes, il se tourne encore plus résolument vers le surnaturel: "il la quitta pour Rose-Mary, à qui sa mère, qui était fée, avait donné un globe cristallin de béryl comme gage de sa pureté ${ }^{11}$. Mais sitôt cette pureté compromise par les soins du poète, le globe se trouble et la belle déflorée le brise dans un accès de fureur, ce qui cause sa mort. Dans un effort de synthèse entre le monde magique et le monde réel, le poète a recours à la magie de son propre verbe pour trouver une compagne selon ses vœux:

Alors il aima Lilith, la première femme d'Adam, qui ne fut pas créée de l'homme. Elle ne fut pas faite de terre rouge, comme Eve, mais de matière inhumaine; elle avait été semblable au serpent, et ce fut elle qui tenta le serpent pour tenter les autres. Il lui parut qu'elle était plus vraiment femme, et la première, de sorte que la fille du Nord qu'il aima finalement dans cette vie, et qu'il épousa, il lui donna le nom de Lilith ${ }^{12}$.

La figure de Lilith n'apparaît qu'une fois explicitement dans la Bible, en tant que «créature de la nuit» (Isaïe 34:14). Elle proviendrait d'une

le Des Esseintes de Huysmans et le Charlus de Proust, auteurs de poésies et de contes fantastiques et macabres (Contes d'un buveur d'éther, 1900).

9. M. Viegnes, «De Monelle aux épouses masquées», p. 53.

Io. M. Schwob, Cour double, p. 97.

II. Ibid.

I2. Ibid., p. 97. 
tradition ancienne, remontant jusqu'à une divinité féminine sumérienne, Lilitu, qui réapparaît peut-être dans l'épopée de Gilgamesh sous l'aspect de Lillaka. Selon une hypothèse non avérée, les Hébreux l'auraient annexée à leur univers religieux lors de l'exil à Babylone, et plus tard les Cabbalistes l'auraient utilisée pour expliquer l'une des énigmes de la Genèse, celle des deux récits de la création d'Adam, que l'on attribue à deux sources réunies, l'Elohiste et le Yahviste. Il est dit dans le premier récit que Dieu crée l'homme "mâle et femelle»; dans le second récit, seul l'Adam mâle est créé, et Eve est tirée de l'une de ses côtes. Il y a donc place pour une première créature femelle, qui aurait été créée en même temps que le mâle et sur un pied d'égalité. Selon un texte cabaliste, l'Alphabet de Ben-Sirah, Lilith aurait même voulu occuper la position supérieure pendant l'acte sexuel, et devant le refus d'Adam, qui entendait lui imposer la position du missionnaire pour affirmer son statut dominant, Lilith se serait révoltée, aurait quitté le Paradis et se serait mêlée à la cohorte des anges déchus. Selon certaines versions, c'est même elle qui se cachait sous les traits du Serpent, Mélusine avant l'heure, pour tenter le nouveau couple et se venger ainsi d'Adam, de sa rivale Eve et du Créateur lui-même ${ }^{13}$.

Cette figure, qui est l'un des archétypes de l'imaginaire féminin fin-de-siècle, se retrouve également dans la peinture préraphaélite, chez Rossetti et John Collier, et elle apparaît jusque dans la littérature $\mathrm{du} \mathrm{XX}^{\mathrm{e}}$ siècle; on a pu voir une filiation entre Lilith et la Lolita de Nabokov. Ce qui caractérise Lilith, outre son indépendance farouche vis-à-vis de l'homme, qu'elle domine dans le rapport érotique, c'est son éternelle jeunesse associée à une révolte contre l'ordre établi. En ce sens, et malgré leur absence de perversité, beaucoup de personnages féminins chez Schwob sont des avatars de cette "première femme». C'est le cas de Monelle, la prostituée adolescente de Schwob, qui prêche un "évangile de la pitié»; Le Livre de Monelle (1894) la montre à ce point remplie de compassion pour les enfants, éternelles victimes d'un monde d'adultes cruel et corrompu, qu'elle les soustrait à leurs familles et à la société pour les rassembler dans «la maison où l'on joue», définie comme «une prison où l'on enfermait les innocents pour les empêcher de souffrir, un hôpital où l'on guérissait du travail de la vie». Celle en qui Mario Praz voyait

13. Voir P. Brunel (dir.), Dictionnaire des mythes féminins et dans ce volume l'article de J.-C. Mühlethaler. 
"una Beatrice decadente» ${ }^{14}$ peut aussi apparaître comme une fée protectrice ou une sainte paradoxale. On la retrouve, dans un autre recueil de Schwob, Le Roi au masque d'or, sous les traits de la jeune Maïe, une actrice adolescente qui a recueilli un orphelin bossu, encore plus jeune qu'elle. Elle se confectionne un habit pour une pièce dans laquelle elle a déjà joué, et qui s'intitule "Le Pays bleu». Le narrateur adulte, qui la regarde avec les yeux d'Humbert Humbert pour Lolita, visite fréquemment le taudis qu'elle occupe avec son protégé, jusqu'au jour où il trouve l'endroit désert, avec un écriteau disant: "Partis pour le pays bleu».

On trouve là réunis deux traits de cet imaginaire de la femme, la totale indépendance, et l'attirance pour un univers parallèle, magique ou imaginaire, qui semble être son véritable Heimat: le pays bleu des rêves. Ces deux traits sont réunis dans une réécriture parodique de «La Belle au bois dormant", qui fait partie du recueil Les Oiseaux bleus de Catulle Mendès, publié en 1888. Comme le fait remarquer Jean de Palacio, «le sommeil de la Belle au bois dormant semble avoir fasciné les générations fin-de-siècle» ${ }^{15}$; en effet, il ne recense pas moins de dix variations sur ce thème, et rappelle que Théodore de Banville, cherchant un sujet pour une ouvre lyrique en collaboration avec Jules Massenet, écrit au compositeur «le célèbre conte nous donnerait tout ce que nous cherchons " ${ }^{16}$. "Tout ce que nous cherchons»: un lieu de transition, un sas si l'on peut dire, entre la vie réelle et cette "seconde vie" qu'était le Rêve selon Nerval. Et c'est dans la demeure d'Hypnos que s'effectue le passage: le potentiel mythopoétique du sommeil paraissait logiquement illimité pour ces rêveurs fin-de-siècle. Des rêveurs assez sarcastiques tout de même: le conte de Mendès, qui s'intitule "La Belle au bois rêvant", se présente comme la véritable histoire de cette princesse et le narrateur reproche à Perrault de «ne pas relater les choses exactement de la façon qu'elles s'étaient passées dans le pays de la féerie » ${ }^{17}$. Si lui peut le faire, c'est qu'il a le privilège de posséder un rouet magique, offert par une vieille femme de ses connaissances qui est sans doute une fée à la retraite; en tournant, ce rouet, tel un gramophone magique, restitue la version

I4. M. Praz, La Carne, la Morte e il Diavolo nella letteratura romantica, p. 376.

15. J. de Palacio, Les Perversions du merveilleux, p. 153. Voir également l'analyse privilégiée, selon la psychologie des profondeurs de Jung, que Marie-Louise von Franz consacre à ce conte dans La Femme dans les contes de fées.

16. J. de Palacio, Les Perversions du merveilleux, p. 142.

17. C. Mendès, Les Oiseaux bleus, p. 52. 
"originale» du conte ${ }^{18}$. Nous y retrouvons le jeune et vaillant prince qui, après avoir bravé tous les périls, éveille enfin la belle endormie et lui dit:

Si vous daignez ne pas repousser mes vœux, je vous donnerai tout mon cœur, comme un autre royaume dont vous serez la souveraine, et je ne cesserai jamais d'être l'esclave reconnaissant de vos plus cruels caprices.

- Ah! quel bonheur vous me promettez!

- Levez-vous donc, chère âme, et suivez-moi.

- Vous suivre? déjà? Attendez un peu. Il y a sans doute plus d'une chose tentante parmi tout ce que vous m'offrez, mais savez-vous si, pour l'obtenir, il ne me faudrait pas quitter mieux? [...] Je dors depuis un siècle, c'est vrai, mais depuis un siècle, je rêve. Je suis reine aussi, dans mes songes, et de quel divin royaume! [...] Pour ce qui est de l'amour, croyez bien qu'il ne me fait pas défaut; car je suis adorée par un époux plus beau que tous les princes du monde et fidèle depuis cent ans. Tout bien considéré, monseigneur, je crois que je ne gagnerais rien à sortir de mon enchantement; je vous prie de me laisser dormir" [...] Le Prince s'éloigna fort penaud. Et depuis ce temps, grâce à la protection des bonnes fées, personne n'est venu troubler dans son sommeil la «Belle au bois rêvant" ${ }^{19}$.

Outre le pur plaisir de la parodie, cette réécriture affirme aussi, au second degré, la supériorité du rêve sur le réel, ce réel fût-il le monde déjà transfiguré du conte de fées. La princesse de Catulle Mendès choisit délibérément ce que Gérard Peylet nomme le "contre-monde » ${ }^{20}$; il ne s'agit plus d'un simple "épanchement du songe dans la vie réelle», mais d'une suprématie de ce «merveilleux au carré» qu'est le rêve dans

I8. Il faut se rappeler que l'invention simultanée du gramophone, ou phonographe, par le poète français Charles Cros et l'ingénieur américain Thomas Edison, en 1879, causa une émotion considérable dans les milieux artistiques et littéraires, où la possibilité d'entendre à volonté la voix d'une personne, fût-elle décédée, relevait autant de la science que de la nécromancie. C'est ce qui explique que Villiers de l'Isle-Adam fasse d'Edison le protagoniste de son roman L'Eve future (1889), dans lequel le sorcier de Menlo Park crée une "andréide", un robot féminin, destiné à suppléer aux imperfections de la femme dans sa version organique. Jules Verne s'inspire aussi de cette invention, encore une fois en relation avec le féminin, dans Le Château des Carpathes. Sur le motif croisé du rouet et de la magie de la bobine de film, voir aussi dans ce volume l'article de D. Haase.

19. Ibid., p. 58-59.

20. G. Peylet, La Littérature fin de siècle de 1884 à 1898, p. 134. 
un rêve. Le corollaire implicite de cette suprématie est la misère ontologique du monde dit «réel» à l'époque de l'industrie et du mercantilisme triomphant. C'est là un thème qui revient souvent, dans le texte ou dans le paratexte du conte merveilleux fin-de-siècle. Dans la préface à son recueil Princesses d'ivoire et d'ivresse, Jean Lorrain écrit:

Comme je plains au fond de moi les enfants de cette génération, qui lisent du Jules Verne au lieu de Perrault, et du Flammarion au lieu d'Andersen! ${ }^{21}$

Très peu sensible à ce que Todorov appellera le «merveilleux scientifique", l'auteur délicat et pervers de Monsieur de Phocas préfère l'audelà des légendes à l'en-deçà du monde contemporain, qui pour lui est beaucoup plus «irréel», avec ses valeurs bourgeoises et triviales, que le Wonderland. On peut voir une autre réécriture de "La Belle au bois dormant" dans un conte intitulé "Mandosiane captive», du même recueil $^{22}$. La belle princesse Mandosiane est âgée de six cents ans, mais elle n'a pas pris une seule ride, car elle n'est qu'une image brodée sur velours avec un visage et des mains de soie peinte, "née du rêve et du travail obstiné» de nonnes du Moyen Age ${ }^{23}$. Longtemps exposée aux regards admiratifs lors de processions princières, elle dort maintenant d'un poussiéreux sommeil au fond d'une crypte. Elle mourrait d'ennui si elle pouvait mourir, et maudit le sort qui l'a faite captive de cette tapisserie. C'est alors qu'une souris rouge - peut-être une fée? - vient lui faire une proposition intéressante:

2I. J. Lorrain, Princesses d'ivoire et d'ivresse, p. 2. Toutefois, un peu plus loin, l'auteur des Histoires de masques jette bas le sien et fait entrevoir à son lecteur le détournement caractéristique qu'il va faire subir à ce merveilleux de bonne famille: «Il faut donc aimer les contes, il faut s'en nourrir et s'en griser comme d'un vin peu dangereux et léger, mais dont la saveur âcre sous un faux goût de sucre insiste et persiste, et c'est cette saveur-là qui, le repas fini, enchante le palais et permet au convive écœuré de la table parfois d'y demeurer» (ibid., p. 3).

22. Ce recueil, dans l'édition précitée, se divise en quatre «sous-recueils»: un premier du même titre, puis Princesses d'ambre et d'Italie, Masques dans la tapisserie, où figure "Mandosiane captive», et Contes de givre et de sommeil.

23. A la fois muse et œuvre d'art, cette figure convoque et les sources médiévales de «La Belle au bois dormant» et la dimension auto-réflexive des contes précieux du XVII ${ }^{\mathrm{e}}$ siècle français (voir l'article de $\mathrm{S}$. Ballestra-Puech dans le présent volume). 
Ce n'est pas une vie que la tienne. Tu n'as jamais vécu, même au temps où tu resplendissais sous le ciel bleu des fêtes carillonnées, acclamées par l'ivresse des foules, et maintenant tu vois, c'est l'oubli, c'est la mort. Si tu voulais, avec mes dents pointues je déferais un à un les points de soie et de cordonnet d'or qui te tiennent fixée depuis six cents ans immobile dans ce velours [...] tu te feras habiller chez les plus grands faiseurs, on te prendra pour la fille d'un banquier et tu épouseras pour le moins un prince français [...] laisse-moi te délivrer! tu révolutionneras le monde! ${ }^{24}$

On comprend tout de suite qu'il faudrait se méfier de cette souris très moderne, qui parle presque un langage d'impresario pour top model et propose à la princesse médiévale une existence de people; mais la naïve Mandosiane accepte, et sitôt délivrée par les dents du rongeur de la tapisserie où elle avait toujours vécu, elle tombe en lambeaux et en poussière sur le sol de la vieille crypte. "Ainsi mourut la princesse Mandosiane pour avoir écouté les insidieux conseils d'une petite souris» ${ }^{25}$. On pourrait voir dans le destin funeste de Mandosiane le châtiment de la femme qui cède à la tentation de s'émanciper des rôles prescrits pour elle, notamment des plus prestigieux, tels ceux de muse et d'icône. Si l'on tient compte de la posture littéraire générale de Lorrain, le message le plus clair est que le merveilleux traditionnel peut s'accommoder à bien des sauces, même à des ragoûts un peu pervers, mais il ne saurait survivre si l'on cherche à l'affubler des oripeaux vulgaires de l'époque contemporaine.

C'est d'ailleurs le même message que l'on trouve dans «La dernière Fée» de Catulle Mendès, un autre conte du recueil Les Oiseaux bleus ${ }^{26}$ : la fée Oriane ne comprend pas ce qui lui arrive. Là où naguère régnaient les belles ramures de la forêt de Brocéliande, s'étend maintenant «une vaste plaine, avec des bâtisses éparses sous un ciel sali de noires fumées» ${ }^{27}$; un lézard qui passe par là lui explique que les hommes ont dévasté la forêt "pour qu'on pût bâtir des maisons et pour ouvrir un passage à d'affreuses machines soufflant des vapeurs et des flammes» ${ }^{28}$. Désespérée, Oriane,

24. Ibid., p. 181.

25. Ibid., p. 182.

26. Sur l'importance du recueil dans la poétique de ce type de contes, rédigés souvent par des poètes, voir l'étude éclairante de B. Vibert, Poète, même en prose.

27. C. Mendès, Les Oiseaux bleus, p. 248.

28. Ibid., p. 249. 
qui est une bonne fée, se console en constatant que ses pouvoirs sont intacts, et elle avise une jeune fille mélancolique qui soupire en l'absence de celui qu'elle aime. Aussitôt, la dernière fée, comme une bonne marraine qu'elle est, lui propose de le faire apparaître sur-le-champ devant elle, ce que la jeune fille accepte avec gratitude. Mais au lieu d'un jeune premier à la mine fraîche et avenante, Oriane voit surgir « un fort laid personnage, vieillissant, l'œil chassieux, la lèvre fanée [qui] portait, dans un coffret ouvert, tout un million de pierreries»; et la jeune fille court à lui et le «baise sur la bouche d'un passionné baiser» ${ }^{29}$.

Triomphe du réel le plus sordide sur le rêve, de l'argent sur l'idéal. On voit là l'une des fonctions, accusatoire, du conte merveilleux fin-desiècle, dont on ne peut dire si sa fonction ultime est bien «émancipatrice» comme le voudrait Jack Zipes ${ }^{30}$, ou s'il ne fait qu'exprimer en mode décalé un constat désespéré sur la modernité et l'Entzauberung. Mais revenons aux variations sur «La Belle au bois dormant»: affirmer la toute-puissance du Rêve peut sembler l'antidote à la misère du temps présent, ou du Temps tout court. Néanmoins les choses ne sont pas si simples. Le pouvoir féminin, dans cet imaginaire fin-de-siècle, connaît aussi ses limites, et la mort en est une. Témoin la fin de ce poème de Henri de Régnier, autre réécriture du conte : «Et la Belle s'endormit / Et le chevalier ne vint pas / Et la belle mourut" ${ }^{31}$. A côté de ce rire fin-desiècle volontiers parodique et cynique, qu'a étudié Daniel Grojnowski ${ }^{32}$, on trouve aussi une profonde mélancolie dans ces contes dont l'esthétique participe du symbolisme et de la décadence. La mort des princesses ou des fées n'y est pas seulement une fatalité individuelle, mais aussi une fatalité de l'Histoire. Les temps modernes, avec leur matérialisme triomphant, répètent des époques antérieures qui ont été néfastes aux médiatrices de l'invisible, comme on le voit dans "Oriane vaincue», un

29. Ibid., p. 255.

30. "A quelques exceptions près, les contes émancipateurs sont généralement écrits avec astuce, humour et originalité; ils réussissent à stimuler la recherche de solutions aux réalités qu'ils dénoncent dans le conformisme ambiant." (J. Zipes, Les Contes de fées et l'art de la subversion, p. 312). Un tel optimisme cadrerait mal avec l'ethos de la fin de siècle. Sur l'usage subversif du conte de fées au cours de l'histoire, voir également J. Mainil, Madame d'Aulnoy et le Rire des fées et E. W. Harries, Twice upon a Time.

31. H. de Régnier, Poèmes anciens et romanesques, cité par J. de Palacio, Les Perversions du merveilleux, p. 257.

32. D. Grojnowski, Aux commencements du rire moderne. 
autre conte de Princesses d'ivoire et d'ivresse; la belle fée, qui a les allures séductrices d'une Circé ou d'une Calypso, a attiré à sa caverne maints chevaliers fous d'amour, et les a plongés dans un sommeil magique où chacun peut rêver qu'il est auprès d'elle:

Captive de leurs désirs comme ils sont, eux, captifs de sa beauté, Oriane se cambre et se meut lentement sous sa chevelure de clair de lune, s'étire voluptueuse, puis se penche éblouie vers un petit miroir ovale qu'elle tient d'une main; opale mystérieuse au fond de laquelle apparaît et s'évanouit tour à tour le visage de prière de chacun des guerriers ${ }^{33}$.

Variante de la belle endormie, ce sont ici les chevaliers qui vivent dans le sommeil et le rêve. Mais Oriane sait qu'elle va bientôt mourir, car une vision lui a montré un jeune chevalier, prévenu contre ses charmes - au double sens du terme - par des moines austères qui l'ont envoyé pour délivrer les chevaliers et tuer celle qu'ils voient comme une démoniaque créature:

Les temps étaient révolus, elle était vaincue d'avance. C'était le Christ qui marchait avec cet enfant, le Christ ennemi de la joie, de la volupté et de l'amour. C'est lui qui avait suscité contre elle ce bourreau à face d'archange et voilà que deux larmes perlaient aux yeux pâles de la fée et que se ternissait tout à fait l'éclat de son miroir ${ }^{34}$.

Ironie finale, lorsque ce héros chrétien fait irruption dans la caverne d'Oriane, il tire effectivement, par une formule sacrée, les chevaliers chrétiens de leur sommeil magique, mais c'est pour voir se lever des morts vivants qui se décomposent et tombent en morceaux à peine debout. La fée elle-même, subitement vieillie et mourante, interpelle le jeune chevalier:

O malheureux enfant, la dernière illusion qu'avaient encore les hommes, fleurissait dans ces bois, et c'est toi qui l'as tuée ${ }^{35}$.

33. J. Lorrain, Princesses d'ivoire et d'ivresse, p. 187.

34. Ibid., p. 189-190.

35. Ibid., p. 192. On retrouve là un motif qui traverse le XIX ${ }^{\mathrm{e}}$ siècle, celui du conflit entre paganisme et christianisme, avec une sympathie affichée pour le premier, comme il apparaît notamment dans les fictions sur le retour de Vénus chez Eichendorff, Mérimée et Heine. Dans ce conte, le Moyen Age est l'époque qui proclame que «le 
Ce monde de l'illusion, dont le féminin semble toujours être si proche, n'est pas toujours univoque: dans ce conte de Lorrain, il est assez ambigu, puisque les amoureux d'Oriane semblent enfermés dans un rêve de désir, et le texte ne dit pas que leur désir soit satisfait, même de manière onirique, ou "fantasmatique" comme dira plus tard Freud. Mais les sortilèges d'Hypnos peuvent même devenir redoutables, et le rêve virer au cauchemar. On en voit un exemple dans deux contes de Princesse d'ivoire et d'ivresse, qui sont en fait deux variantes d'un même récit, reprenant le schéma classique des mythes, avec l'hubris et son châtiment. Le miroir retrouve son statut d'accessoire obligé dans ces contes qui mettent en scène une princesse amoureuse de sa propre beauté. "La Princesse au sabbat» nous présente Ilsée qui «n'aimait que les miroirs et les fleurs [...] plus amoureuse d'elle-même que ne le fut jadis Narcissus, elle s'imaginait être la filleule des fées et sa délicate petite personne lui inspirait un respect infini. Or les fées lui jouèrent un tour ${ }^{36}$.

Ce mauvais tour, c'est le même que jouent les sorcières à Illys, princesse d'Egypte, dans «La princesse aux miroirs»: belle comme une déesse, celle-ci veut conclure un pacte avec les sorcières lybiques: elle se livrera corps et âme toute une nuit à elles au cours de leur sabbat dans les montagnes du désert, en échange d'un philtre qui lui donnera la jeunesse éternelle et préservera sa beauté pour toujours. Ce philtre est chèrement payé, car la princesse vit une nuit de cauchemar ${ }^{37}$, mais en outre c'est un marché de dupes, comme elle s'en rend compte le lendemain matin, en se réveillant dans son palais:

Les miroirs ne lui montrèrent plus jamais le péché de sa beauté. Vainement chercha-t-elle ses yeux et son sourire. Illys ne retrouva

grand Pan est mort»: c'est l'une des nombreuses facettes de ce millénaire qui suscite beaucoup de fantasmes dans la littérature et l'art du XIX ${ }^{\mathrm{e}}$ siècle. Voir à ce sujet S. Bernard-Griffiths, P. Glaudes, B. Vibert (éds), La Fabrique du Moyen Age.

36. J. Lorrain, Princesses d'ivoire et d'ivresse, p. 25-28.

37. Les descriptions de Lorrain anticipent les délires de Lovecraft, tout en portant le cachet typique de la gynophobie fin de siècle que l'on retrouve dans certains tableaux contemporains de Félicien Rops ou Gustave Adolph Mossa. Témoin ce détail de la scène du sabbat, avec des sorcières "qui ont des trompes d'éléphant au milieu de leur visage et cette trompe s'enroule autour de leurs jambes et vient flairer, à la place de leur sexe, une étrange petite tête de mort» (p. 53). Sur ce type d'iconographie, voir l'étude classique de B. Dijkstra, Idoles de la perversité. 
jamais son image, elle l'avait laissée au Sabbat. Les sorcières d'Egypte lui jouèrent ce tour pour la punir de son orgueil ${ }^{38}$.

La punition est subtile, car le texte ne dit pas que les sorcières n'ont pas effectivement immortalisé la beauté de la princesse; mais elles lui ont interdit à tout jamais d'en jouir. Le miroir, instrument du péché, est aussi l'instrument de la punition, comme dans le conte précédent, dont la conclusion est quasiment identique:

La princesse Ilsée ne retrouva jamais son image; elle l'avait laissée au Sabbat: les fées lui jouèrent ce tour pour la punir de son orgueil ${ }^{39}$.

Lorrain a peut-être emprunté le nom de cette princesse à Schwob. Dans l'un des contes enchâssés du Livre de Monelle, la narcissique Ilsée voit son double sortir du miroir pour l'emporter dans la mort. D'après l'un des premiers commentateurs de Schwob, Georges Trembley, «le miroir [...] apparaît moins comme le gage d'une réalité au-delà de ce monde, que comme un piège ${ }^{40}$.

Certes, le miroir est l'instrument du narcissisme, qui appelle la justice poétique du châtiment par les forces surnaturelles, mais il fonctionne aussi chez Lorrain comme le seuil d'un autre monde. Bertrade, la jeune et pure héroïne de "La Princesse sous verre», du recueil Contes de givre et de sommeil, ne peut être soupçonnée du moindre orgueil. Et pourtant:

Dans toute la nature elle ne paraissait aimer que les reflets. L'eau aussi l'attirait [...] elle aimait au crépuscule à s'attarder aux bords glacés des sources et dans le brouillard fiévreux des étangs mais à tout au monde elle préférait les interminables et silencieuses haltes devant l'étain figé des glaces ${ }^{41}$.

Mais c'est parce que l'orpheline, inconsolable de la mort de sa mère, cherche dans tous ces reflets un seuil sur l'au-delà :

L'âme de sa mère semblait l'y retenir, remontée des ténèbres à la surface équivoque des miroirs ${ }^{42}$.

38. Ibid., p. 54-55.

39. Ibid., p. 33.

40. G. Trembley, Marcel Schwob, faussaire de la nature, p. 70.

4I. J. Lorrain, Princesses d'ivoire et d'ivresse, p. 224.

42. Ibid. 
Même si ce désir est plus pur que celui des princesses narcissiques, il participe d'une dimension négative, ou du moins dangereuse, de l'imaginaire. En se détournant ainsi de la vie pour ce monde de reflets, la princesse perd toutes ses forces vitales et sombre dans un si profond sommeil qu'on la croit d'abord morte, pour comprendre bientôt qu'elle «n'était qu'endormie, mais de quel étrange et lugubre sommeil!». «Elle ne vivait ni ne mourait ${ }^{43}$; espérant la réveiller, on la promène en procession à travers tout le pays, dans une châsse de verre, comme une sainte. Ce sera en vain. Autre réécriture de "La Belle au bois dormant», en mode sombre. Mais cette histoire tragique se termine, de façon improbable, comme une hagiographie: la princesse endormie meurt sans s'être réveillée, mais après avoir sauvé l'âme d'un seigneur criminel. Le sortilège du sommeil est vaincu non par l'amour humain, mais par l'Agapè qui ouvre sur l'éternité. Fin positive, mais le cheminement, dans ce récit qui semble imité de La Légende dorée, est passablement long. De même, la victoire de Lusignan sur le maléfice qui avait transformé Mélusine en Hydre est chèrement acquise, puisqu'il doit avaler à trois reprises la bave et le venin du monstre, avant que celui-ci ne reprenne la forme beaucoup plus aimable qu'il avait avant que les fées ne lui fassent subir l'ignoble métamorphose. Dans cette version de la légende médiévale, Lorrain ne présente pas le maléfice comme une punition de l'orgueil, mais comme un effet de la jalousie d'un monde féerique plutôt ténébreux:

Et les fées jalouses l'ont changée en serpent; son impérieuse beauté, qui charmait les oiseaux du ciel et les bêtes errantes des bois, épouvante aujourd'hui la solitude [...] Où cela? Très loin et tout près d'ici, dans le pays des fées, qui veillent invisibles sur leur prisonnière, dans les landes d'or $[\ldots]$ que vous avez cent fois traversées sans soupçonner que les malignes dames riaient dans la broussaille, assises en cercle autour de vous ${ }^{44}$.

Le plus terrible, avec les fées et autres figures du féminin magique, ce n'est pas qu'elles soient malignes, cruelles, perverses, mais plutôt qu'elles se meurent. Le conte merveilleux fin-de-siècle, derrière ses jeux d'écriture parodiques, ses détournements érotiques ou macabres, qui préfigurent

43. Ibid., p. 225-226.

44. "Mélusine enchantée", dans Masques dans la tapisserie (Princesses d'ivoire et d'ivresse), p. 172. 
des réécritures contemporaines ${ }^{45}$, est traversé par une angoisse unique, mais profonde et sans remède, celle du désenchantement du monde, qui vide la réalité moderne de tout mystère. Or, comme l'affirmait assez justement le pompeux Péladan dans son essai Comment on devient fée, "la première condition du pouvoir féérique, c'est son mystère " ${ }^{46}$. Ce personnage dérisoire, mage autoproclamé de cette contre-culture à l'apogée du positivisme, a compris qu'un monde sans mystère est comme la «terre gaste» de la légende du Graal, un Wasteland aussi désolé qu’un monde sans amour.

Michel Viegnes

Université de Fribourg

45. Comme celles d'Angela Carter dans The Bloody Chamber and Other Stories. Voir l'article de M. Hennard Dutheil de la Rochère dans ce volume.

46. Cité par J. de Palacio, Les Perversions du merveilleux, p. 67. 


\section{BIBLIOGRAPHIE}

\section{Sources}

Barbey d’Aurevilly, Jules, Les Diaboliques, Paris, Gallimard/Folio classique, 2000 [1874].

Carter, Angela, The Bloody Chamber and other stories, Harmondsworth, Penguin Books, 1979.

Huysmans, Joris Karl, Là-bas, Paris, Gallimard/Folio, 1999 [1891].

Lorrain, Jean, Princesses d'ivoire et d'ivresse, Paris, Ollendorf, 1902.

Mendès, Catulle, Les Oiseaux bleus, Paris, Nouvelles Editions Séguier, «Bibliothèque décadente», 1993 [1888].

Mirbeau, Octave, Le Jardin des supplices, Paris, Gallimard/Folio, 1999 [1898].

Régnier, Henri de, Poèmes anciens et romanesques, Paris, Librairie de l'Art Indépendant, 1890.

Sсншов, Marcel, Cour double, Paris, Gallimard «L'Imaginaire», 1997 [1892].

\section{Travaux}

Bernard-Griffiths, Simone, Glaudes, Pierre, Vibert, Bertrand (éds), La Fabrique du Moyen Age, Paris, Champion, 2006.

Brunel, Pierre (dir.), Dictionnaire des mythes féminins, Monaco, Editions du Rocher, 2002.

DéCaudin, Michel, "Salomé: un mythe fin-de-siècle", Comparative Literature Studies, 4/1-2 (1967), p. 109-117.

Dijnstra, Bram, Idoles de la perversité. La Femme fatale dans la culture fin de siècle, Paris, Seuil, 1990.

Franz, Marie-Louise von, La Femme dans les contes de fées (trad. Francine Saint-René Taillandier), Paris, La Fontaine de pierre, 1984. 
Grojnowski, Daniel, Aux commencements du rire moderne. L'Esprit fumiste, Paris, José Corti, 1997.

Harries, Elisabeth Wanning, Twice upon a Time. Women Writers and the History of the Fairy Tale, Princeton, Princeton Univ. Press, 2003.

Mainil, Jean, Madame d'Aulnoy et le Rire des fées. Essai sur la subversion féérique et le merveilleux comique sous l'Ancien Régime, Paris, Kimé, 2001.

Palacio, Jean de, Les Perversions du merveilleux, Paris, Séguier, 1993.

Peylet, Gérard, La Littérature fin de siècle de 1884 à 1898. Entre décadentisme et modernité, Paris, Vuibert, 1993.

Praz, Mario, La Carne, la Morte e il Diavolo nella letteratura romantica, Firenze, Adriatice Ed., 1948.

Trembley, Georges, Marcel Schwob, faussaire de la nature, Genève, Droz, 1969.

Vibert, Bertrand, Poète, même en prose. Le Recueil de contes symboliste 1890-1900, Saint-Denis, Presses Universitaires de Vincennes, «L'Imaginaire du texte», 2010.

Viegnes, Michel, "De Monelle aux épouses masquées: le thème féminin dans l'œuvre de Marcel Schwob", Romance Notes, XVII, 1, (automne 1986), p. 53-59.

—, "Gynophobia: la peur du féminin dans le récit fantastique», Cahiers du GERF, 6 (1999), p. 81-97.

Zipes, Jack, Les Contes de fées et l'art de la subversion, trad. F. Ruy-Vidal, Paris, Payot, 2007 [1983]. 